\title{
Phosphate ions alter the binding of daptomycin to living cell membranes
}

\author{
Lindsey N. Miller and Tessa R. Calhoun* \\ Department of Chemistry, University of Tennessee, Knoxville \\ E-mail: trcalhoun@utk.edu
}

\begin{abstract}
Advancements in antibiotic drug design are often hindered by missing information in how these small molecules interact with living cells. The antibiotic, daptomycin, has found clinical success, and emerging resistance, but a comprehensive picture of its mechanism of action has remained elusive. Using the surface-specific spectroscopy, second harmonic generation, we are able to quantitatively assess the binding of daptomycin to living cell membranes without the addition of exogenous labels. Our results reveal similar binding affinities for both gram-positive and gram-negative bacteria studied, including E. coli. More importantly, we show that phosphate ions influence the binding of daptomycin to the gram-positive bacterium E. faecalis. The role of environmental phosphate has not previously been considered in any proposed mechanism and its implications are expected to be important in vivo.
\end{abstract}

\section{Introduction}

The World Health Organization has listed multi-antibiotic resistance as one of the biggest global health threats in $2020 .{ }^{1}$ In 2019, the Centers for Disease Control and Prevention 
(CDC) reported that over 2.8 million new antibiotic-resistant infections are being documented every year, resulting in more than 35,000 yearly deaths in the United States alone. $^{2}$ The greatest threats arise from the growing number of multidrug-resistant bacterial strains. ${ }^{2-6}$ Of these strains, two of the gram-positive bacterial species that the CDC ranked as serious threats are vancomycin-resistant Enterococcus faecalis and methicillinresistant Staphylococcus aureus (MRSA). ${ }^{2,3}$ When first discovered over 30 years ago, the antibiotic daptomycin yielded successful results for effectively treating these gram-positive bacterial infections. ${ }^{7-10}$ Considered one of the first antibiotics of its kind, daptomycin is a calcium-dependent cyclical lipopeptide drug that is believed to cause cell death through depolarization upon inserting into gram-positive bacterial membranes. ${ }^{9,11}$ The formation of the nascent drug complex and its interaction with living bacterial membranes is still poorly understood. $^{9-13}$ The need for this information has become more imperative as daptomycinresistant gram-positive strains are emerging quickly with minimal treatment options remaining. ${ }^{10,12-22}$ Considering the impact of various environmental factors on daptomycin's affinity for living bacterial membranes could provide new, crucial information for improved drug development. The work presented examines the initial native daptomycin-membrane adsorption affinities in living bacteria under different environmental conditions by measuring concentration-dependent isotherms using second harmonic generation (SHG), a surfacespecific spectroscopy technique.

Daptomycin activity has been shown to require the presence of calcium ions and negatively charged phospholipids such as phosphatidylglyercol $(\mathrm{PG}$, net charge $=-1)$ and cardiolipin $(\mathrm{CL}$, net charge $=-2)$, the latter of which are found abundantly in gram-positive bacterial lipid-membrane compositions. ${ }^{11,23,24}$ When these two criteria are met, daptomycin is thought to insert its nonpolar tail into the bacterial membrane. However, calcium's role for facilitating daptomycin-membrane activity is still highly debated. ${ }^{12}$ For example, while many have proposed that calcium ions predominately bind to daptomycin to produce an overall positively-charged complex to form, the composition of this complex is still not agreed 
upon. ${ }^{11,23,25-32}$ An alternative theory suggests that $\mathrm{Ca}^{2+}$ ions interact with the bacterial membrane surface to help shield the negative charges of the phospholipids so that daptomycin can insert into the lipid-bilayer. ${ }^{30}$ More so, the daptomycin:calcium (Dap-Ca ${ }^{2+}$ ) interaction could be altered by other seemingly innocuous factors/species lurking within the surrounding environment. Recently, a new mechanism has been proposed in which lipid II, a cell biosynthesis precursor, has been implicated in daptomycin binding. ${ }^{33}$ The roles of other ions found within various aqueous media environments, however, have not been fully explored possibly explaining the lack of consensus regarding this drug's action.

The affinity of daptomycin for the membranes of living bacteria is challenging to determine as the drug's native, and relatively weak, fluorescence in the visible overlaps within the autofluorescence spectral region for bacterial cells. This is often remedied by studies using either model lipid vesicle systems to avoid cellular autofluorescence signals altogether or by using fluorescently-labeled daptomycin with bacterial cells to cause a spectral shift outside of the autofluorescence region and increase the overall quantum yield of the detected molecule. However, the majority of model membrane spectroscopy studies utilized large unilamellar vesicles (LUVs) containing phosphatidylcholine (PC) phospholipids to indicate both fluorescently-labeled and native daptomycin interaction. ${ }^{23,24,34-40}$ The diameter range of an LUV is defined as 100-1,000 nm whereas the diameter of a gram-positive bacterial cell ranges between 1.5 - 2 microns. The size discrepancies between the LUV and a gram-positive bacterial cell could lead to inaccurate representations of the bacterial membrane curvature and possibly alter the drug's true mechanism for how it interacts with living cell membranes. ${ }^{41}$ PC phospholipids are also not native to the lipid compositions of gram-positive bacteria, such as E. faecalis, and could lead to potentially false mechanisms of action for daptomycin. In addition, model membrane studies lose the ability to account for the complex diversity of lipid and protein species found in biological membranes which vary between bacterial strains. This was demonstrated in our previous work where the variance between the lipid-membrane compositions for E. faecalis and S. aureus was observed to yield different dynamics for the 
probe molecule FM 4-64 as it interacted with the membranes over time. ${ }^{42}$

While the fluorescent-labeling approach is considered a standard technique for optical studies, dye molecules can alter molecular interactions which may lead to inaccurate assumptions regarding the native drug mechanisms. ${ }^{43,44}$ One study noted this discrepancy by showing fluorescently-labeled daptomycin rapidly diffusing into Staphylococcus epidermis within minutes as compared to the native daptomycin case which required several hours for diffusion. ${ }^{44}$ As an alternative, SHG is a nonlinear optical phenomena that only generates signal from chromophores in a noncentrosymmetric environment, such as those in a biological membrane. This removes background from autofluorescent sources in cells, such as the metabolites like NADH, ${ }^{45,46}$ and provides a new means to probe the binding of small molecules, such as drugs, to the membranes of living bacterial cells. Over the past few decades, SHG has been utilized to monitor numerous small molecule-membrane interactions in model systems ${ }^{47-59}$ as well as in living cell systems. ${ }^{42,60-68}$

SHG occurs when two photons of frequency $\omega$ are upconverted to a single photon of frequency $2 \omega$ when they interact with a noncentrosymmetric sample. A more detailed explanation of SHG is provided in the SI, but in our sample the molecule being probed generates coherent SHG signal when it satisfies the noncentrosymmetric criteria by embedding into the lipid bilayer. ${ }^{69}$ A significant increase in the generated SHG signal arises when an electronic transition in the molecule of interest is resonant with either the fundamental or second harmonic frequency. In daptomycin, this resonance condition is met by the kynurenine residue. This chromophore has a weak absorption at $380 \mathrm{~nm}$ with a tail that overlaps with the 400 $\mathrm{nm}$ second harmonic of our laser source. Fluorescence studies have shown an increase in fluorescence signal from the kynurenine residue as native daptomycin inserted into model membranes indicating that the kynurenine residue embedded into the membrane where it became sterically restricted. ${ }^{25,35}$

Langmuir adsorption isotherms can be measured using SHG spectroscopy without the addition of exogenous labels to determine and compare daptomycin's binding affinities for 
cell membranes while in various environmental conditions, essentially revealing daptomycin's unaltered initial interactions with living bacterial systems. Before now, past daptomycin isotherm studies required the use of model membrane systems or fluorescent tags. ${ }^{23,37,40,70-73}$ SHG Langmuir adsorption isotherms have been previously employed to assess the binding affinities for different small molecule-membrane systems such as in model membranes ${ }^{74-82}$ and in living cell membranes ${ }^{42,64,65}$ The cartoon schematic in Fig. 1 represents the adsorption

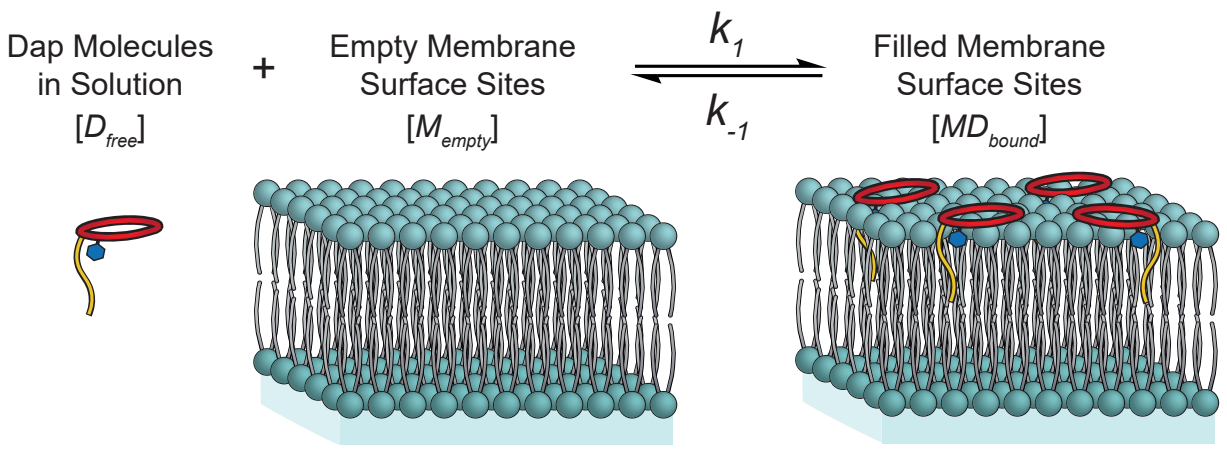

Figure 1: Cartoon schematic of the daptomycin adsorption onto the membrane. Not shown are the $\mathrm{Ca}^{2+}$ ions that are known to be necessary but whose exact role is still debated.

of free daptomycin molecules in solution, $D_{\text {free }}$, onto the empty membrane sites, $M_{\text {empty }}$, and producing daptomycin-bound membrane sites $M D_{\text {bound }}$ that generate the measured SHG signal.

In our experiments, the concentration of daptomycin is increased while monitoring the SHG response to assess the availability of empty membrane sites, $M_{\text {empty }}$. The results can be fit by the equation:

$$
\theta=\frac{\frac{[c]}{K_{d}}}{1+\frac{[c]}{K_{d}}}
$$

where $c$ is the concentration of daptomycin and $\theta$ represents the fraction of $M D_{\text {bound }}$ to $M_{\text {total }}$, such that:

$$
\theta=\frac{M D_{\text {bound }}}{M_{\text {total }}}
$$

$K_{d}$ in Eq. 1 is the dissocation constant $\left(K_{d}=\frac{k_{-1}}{k_{1}}\right)$ and represents the concentration at which half of the membrane sites are occupied. ${ }^{69}$ A smaller $K_{d}$ value corresponds to a stronger 
association with the membrane.

Daptomycin's activity against bacteria has been shown to be impacted by local environment factors including: (1) the ions and molecules in the solvent environment ${ }^{35,83-85}$ and (2) the lipid-membrane composition of the bacterial species. ${ }^{18,20,86-89}$ Here we consider how these factors influence the initial step of daptomycin activity by examining its membrane adsorption/binding. In our experiments detailed below, the daptomycin membrane affinities for the gram-positive bacterial species E. faecalis were determined for solutions with different ionic composition. In order to study the effects arising from the natural membrane composition, we also compared the SHG isotherms for daptomycin with different bacterial species.

\section{Results and discussion}

\section{Ion-Mediated Effects: The Role of Phosphate Ions}

Components found in the surrounding local environment can modify small-molecular interactions at the membrane surface. ${ }^{64,90,91}$ Most biological salines, such as $0.1 \mathrm{M}$ phosphate buffered saline (PBS) and $0.9 \%$ sodium chloride $(\mathrm{NaCl})$, are composed of multiple ionic species with well-defined concentrations. In contrast, bacterial growth in complex media are less defined and contain a wide variety of nutrients such as proteins, lipids, free ions and vitamins. Although, these biological solutions generally have similar $\mathrm{pH}$ values around 7.4, the varying range of ionic and molecular species can dramatically affect how small molecules,

such as daptomycin, interact with bacterial membranes. ${ }^{35,83-85}$ Specific ion effects on membrane processes due to the presence of free ionic species in aqueous environments have been previously investigated using SHG. ${ }^{53,64}$

Figure 2 shows isotherms for daptomycin adsorption onto the membranes of living $E$. faecalis cells in environments with different ionic compositions. The $K_{d}$ values extracted from the fits of the data to the Langmuir equation (Eq. 1) are provided in Table 1. In Fig. 2A, the 

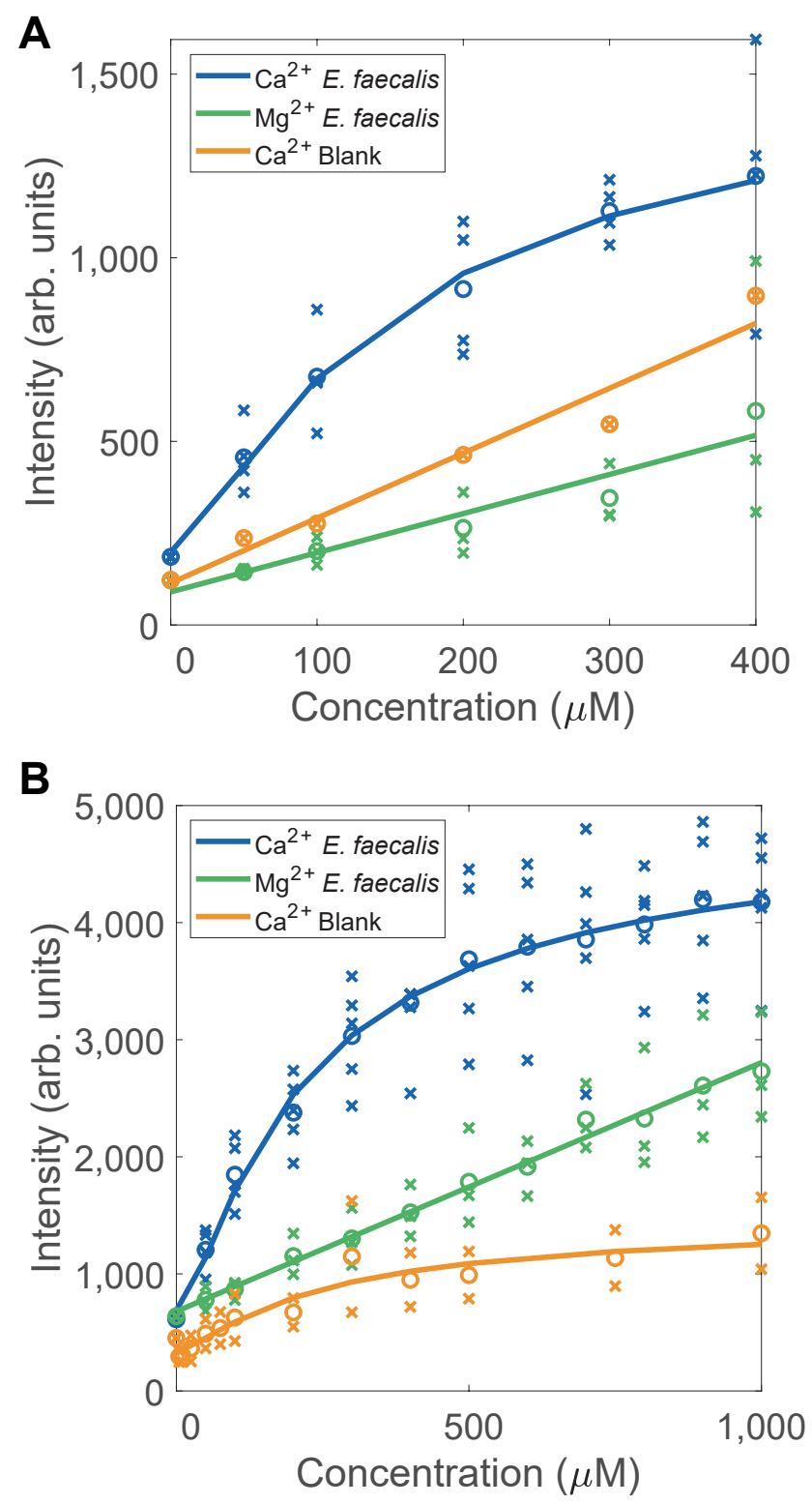

Figure 2: SHG isotherms of daptomycin adsorption studies on E. faecalis cells with a constant 1:8 Dap:Ca ${ }^{2+}$ ratio (-), E. faecalis cells with a constant 1:8 Dap: $\mathrm{Mg}^{2+}$ ratio $(-)$, and blank controls with a constant 1:8 Dap: $\mathrm{Ca}^{2+}$ ratio and no cells present. (-) Cells were suspended in solutions of (A) $0.9 \% \mathrm{NaCl}$ saline and (B) $0.1 \mathrm{M}$ PBS. The individual trials are shown as $\times$. The averages for the curve fits are shown as $\circ$.

cells are suspended in $0.9 \% \mathrm{NaCl}$ saline and the $\mathrm{SHG}$ signal is recorded as the concentration of daptomycin is increased. The blue data points and fit $(-)$ show that the largest signal is observed in $\mathrm{NaCl}$ when calcium ions are also introduced. Here a 1:8 daptomycin:calcium concentration ratio is maintained throughout the experiment. In contrast, significantly less signal is observed if either the cells are absent $(-$, blank control) or if the calcium ions 
are replaced with magnesium ions $(-)$. For the blank control, the linear signal observed with increasing concentration is consistent with hyper-Rayleigh scattering (HRS). HRS is incoherent second harmonic scattering that scales linearly with concentration and arises in this sample from the daptomycin aggregates forming in solution. ${ }^{23,27-32}$ In contrast, the SHG from a single cell is coherent with a forward propagating signal based on the size of the cells. ${ }^{69}$ The SHG signals observed when calcium is replaced by magnesium in the presence of cells is similar to the blank control suggesting that daptomycin is unable to bind to cell membranes under these conditions without calcium present. This is in agreement with other studies that have shown that daptomycin can only bind to membranes in the presence of $\mathrm{Mg}^{2+}$ with significantly higher concentrations. ${ }^{27}$ Overall, our isotherms not only confirm daptomycin's well-known calcium dependency ${ }^{11,23,23,25-30,30-32}$ but also that our measured SHG signals are specific to daptomycin adsorption.

Table 1: Average $K_{d}$ values for the daptomycin binding affinity when using a 1:8 Dap:Ca ${ }^{2+}$ constant ratio with $E$. faecalis cells for $n$ trials.

\begin{tabular}{llll}
\hline Bacterial Species & Saline Environment & $\boldsymbol{n}$ & $\boldsymbol{K}_{d}(\mu \mathbf{M})$ \\
\hline E. faecalis & $0.9 \% \mathrm{NaCl}$ & 4 & $73 \pm 10$ \\
E. faecalis & $\mathrm{PBS}$ & 5 & $104 \pm 9$ \\
E. coli & $\mathrm{PBS}$ & 2 & $92 \pm 24$ \\
S. aureus & $\mathrm{PBS}$ & 3 & $147 \pm 33$ \\
\hline
\end{tabular}

Turning to Fig. 2B, analogous behavior is seen when the experiments are repeated with the E. faecalis cells suspended in PBS. Again, the highest signal is observed when calcium ions are present to facilitate the binding of daptomycin with the cell membranes $(-)$. Looking at the PBS data in more detail, however, reveals multiple key differences. First, the SHG intensities are significantly larger than those from similar concentrations of daptomycin in $\mathrm{NaCl}$. This suggests that in PBS there are either a larger number of surface sites available for daptomycin binding, or that when bound, the daptomycin molecules generate more signal from, for example, more uniform alignment. ${ }^{92}$ A second difference observed for the PBS data is that the blank control $(-)$ is not linear but instead exhibits a Langmuir shape. The Langmuir shape arises from a sharp increase in the initial signal as SHG scales quadratically 
with concentration that begins to level off at higher concentrations as surface sites are filled, preventing additional drug adsorption. In the blank control, there are no cells and thus no membrane surface sites. As such, the plateau observed in the blank control must arise from another limiting reactant.

Calcium and phosphate ions are known to form a precipitate, and a control study was performed in which only calcium was titrated into PBS solution to determine the maximum calcium concentration that could be added to PBS before scatter from these precipitates was significant (Supporting Information, Fig. S1). This control study did not yield a linear nor a Langmuir trend as the calcium concentration was increased again confirming that our SHG isotherm measurements are reporting on daptomycin. The calcium and phosphate control study, however, did exhibit significant scatter for calcium concentrations at and above 10 mM. To stay well under this limit, the maximum calcium concentration was limited to be 8 $\mathrm{mM}$ for all of the SHG isotherm trials to ensure minimal contribution from calcium-phosphate aggregates.

Since daptomycin has been suggested to form aggregate complexes with ionic species, ${ }^{10,31,32,44,52,93}$ dynamic light scattering (DLS) was employed to determine the size of daptomycin aggregates forming within three different solutions. Figure 3 shows the size of the aggregates formed in $\mathrm{NaCl}, \mathrm{PBS}$ and water when 1:0.5 (top) and 1:8 (bottom) concentration ratios of Dap:Ca ${ }^{2+}$ are present. A $1 \mathrm{mM}$ final daptomycin concentration was used throughout the DLS measurements in order to match the highest daptomycin concentration used during the isotherm experiments. When the Dap: $\mathrm{Ca}^{2+}$ is 1:0.5 (Fig. 3 top) the only solution that produces an aggregate species is $\mathrm{NaCl}(-)$ with this complex having a diameter $<10 \mathrm{~nm}$. This species is also present when the relative amount of calcium is increased to a 1:8 $\mathrm{Dap}: \mathrm{Ca}^{2+}$ ratio (Fig. 3 bottom) and a species with a similar size also appears in water solution $(-)$. The most prominent feature in the 1:8 data, however, is a significantly larger aggregate species, approximately $\sim 1 \mu \mathrm{m}$ in diameter, which is only present in the PBS sample (Fig. 3A, - ). The fact that this species only forms in the PBS solution with a relatively large concentra- 
tion of calcium ions suggests that a unique daptomycin complex is formed with calcium that includes an ionic component of PBS.
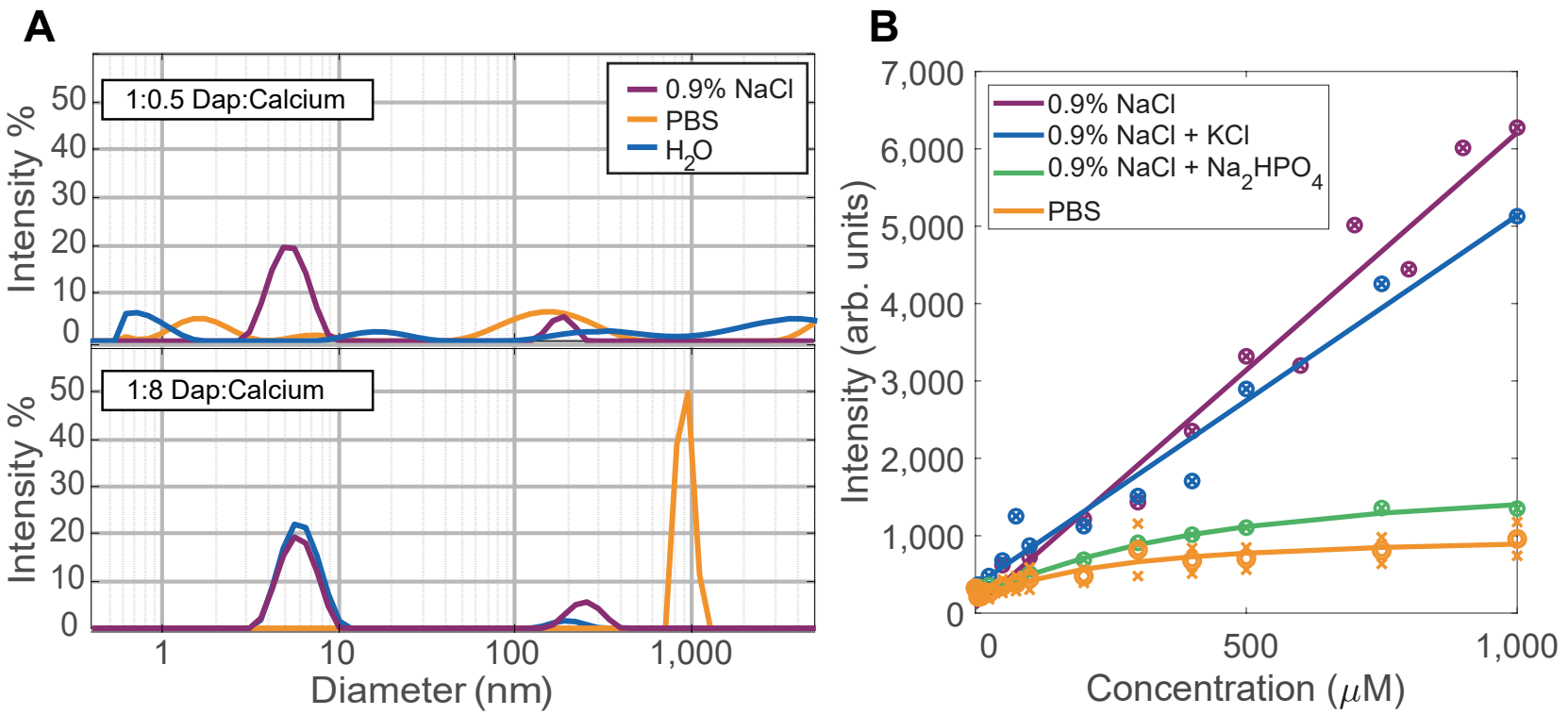

Figure 3: (A) DLS diameter size measurements of Dap: $\mathrm{Ca}^{2+}$ aggregates when in different solutions: $0.9 \%$ $\mathrm{NaCl}$ saline (-), PBS (-) and $\mathrm{H}_{2} \mathrm{O}(-)$. Aggregate sizes are shown for 1:0.5 (top) and 1:8 (bottom) Dap: $\mathrm{Ca}^{2+}$ ratios. Daptomycin concentration was held constant at $1 \mathrm{mM}$ for all DLS trials. (B) Environmental ion effects on 1:8 Dap: $\mathrm{Ca}^{2+}$ ratio daptomycin concentration-dependent SHG isotherms in sterile saline blank controls containing various ion species, such as $0.9 \% \mathrm{NaCl}(-), 0.9 \% \mathrm{NaCl}+1.35 \mathrm{mM} \mathrm{KCl}(-), 0.9 \%$ $\mathrm{NaCl}+5 \mathrm{mM} \mathrm{Na}_{2} \mathrm{HPO}_{4}(-)$ and $0.1 \mathrm{M} \mathrm{PBS}(-)$.

PBS contains the following ionic species: potassium $\left(\mathrm{K}^{+}\right)$, sodium $\left(\mathrm{Na}^{+}\right)$, chloride $\left(\mathrm{Cl}^{-}\right)$, dihydrogen phosphate $\left(\mathrm{H}_{2} \mathrm{PO}_{4}^{-}\right)$and hydrogen phosphate $\left(\mathrm{HPO}_{4}^{2-}\right)$. To determine which of the ionic species found in PBS is contributing to forming the large Dap-Ca ${ }^{2+}$ complex, $0.9 \%$ $\mathrm{NaCl}$ saline solutions were made with the addition of either $\mathrm{KCl}$ or $\mathrm{Na}_{2} \mathrm{HPO}_{4}$ containing 1/2 of the molar equivalents typically used to make 0.1 M PBS. Daptomycin concentrationdependent isotherm control studies were performed without the presence of cells for each of the saline solutions using a constant 1:8 Dap: $\mathrm{Ca}^{2+}$ ion ratio throughout the experiment (Fig. 3B).

Signal resulting from the addition of potassium ions to $0.9 \% \mathrm{NaCl}$ saline (Fig. $3 \mathrm{~B}$, — closely modeled the signal trend seen in the $0.9 \% \mathrm{NaCl}$ saline only sample (Fig. 3B,—). Both of these signals rose linearly with concentration consistent with incoherent HRS. As an aside, the high intensities seen from $\mathrm{HRS}$ in the $\mathrm{NaCl}$ blank prevented higher concentrations 
of daptomycin to be examined for the isotherms presented in Fig. 2A. An extended plot of this data can be found as Fig. S2 in the Supporting Information. Coming back to Fig. 3B, the addition of phosphate ions into $0.9 \% \mathrm{NaCl}$ saline $(-)$ does not display the same linear behavior as the $\mathrm{NaCl}+\mathrm{K}^{+}(-)$but instead closely resembles the signal from the PBS trial $(-)$. These results suggest that free phosphate ions found in the aqueous environment contribute to forming the large and unique daptomycin- $\mathrm{Ca}^{2+}$ complexes. Furthermore, the formation of these clusters directly impacts the binding of daptomycin to the cell membrane as evidenced by the significantly higher SHG signal observed for these conditions (Fig. 2B, -). Environmental phosphate ions are not currently considered to play a role in any of the proposed mechanisms for daptomycin binding ${ }^{11,23,25-32}$ suggesting that these models are still incomplete.

\section{Differences between Bacterial Membrane Compositions}

Bacterial species have unique lipid-membrane compositions which aid in regulating smallmolecule interactions at the membrane surface ${ }^{94-98}$ Daptomycin activity is specific for grampositive bacterial species and has been shown to have no effect on gram-negative species such as E. coli. ${ }^{7,8,99}$ As previously mentioned, daptomycin requires the presence of negatively charged lipid species and has a high affinity for PG phospholipids ${ }^{11}$ which are commonly

found as the major lipid-class in gram-positive bacteria, such as in E. faecalis. ${ }^{100,101}$ Studies have also shown that variations to bacterial lipid-membrane compositions can alter daptomycin behavior. ${ }^{86,89}$ To determine how bacterial phospholipid-membrane compositions affect the initial binding affinity for daptomycin, SHG adsorption isotherms were measured with E. coli and S. aureus while maintaining a constant 1:8 $\mathrm{Dap}: \mathrm{Ca}^{2+}$ ion ratio as the daptomycin concentration was increased. The SHG adsorption isotherms for each bacteria as well as the previous data from E. faecalis are shown in Fig. 4 and the extracted $K_{d}$ are reported in Table 1.

It is immediately apparent that despite being inactive against $E$. coli, daptomycin still 


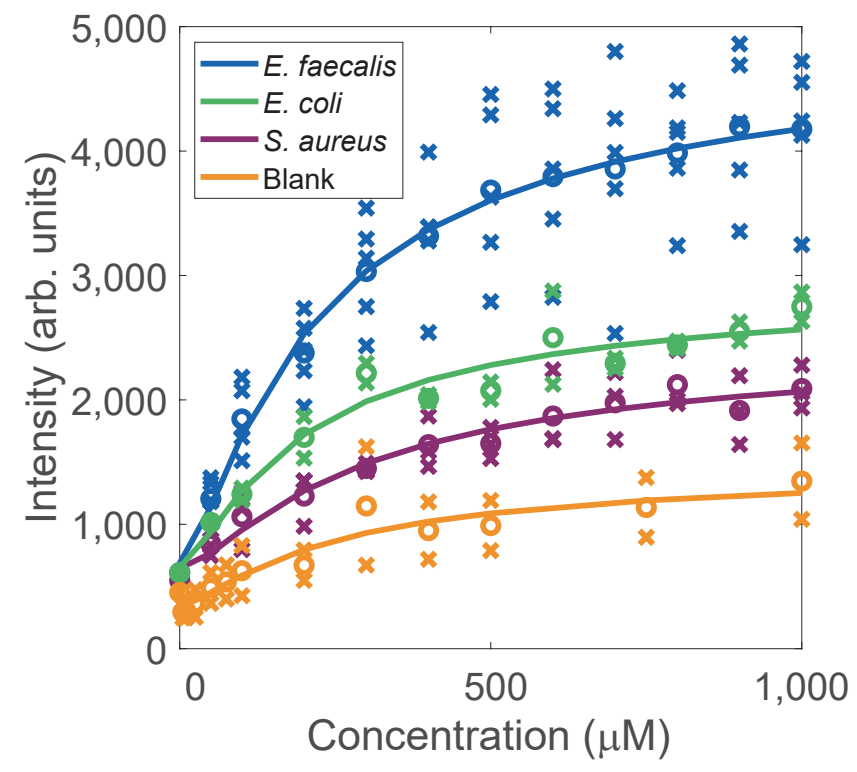

Figure 4: SHG isotherms of initial daptomycin adsorption onto the membranes of different bacterial species. E. faecalis cells $(-)$, E. coli cells $(-)$, S. aureus cells (-) and a comparison to the 1:8 Dap:Ca ${ }^{2+}$ PBS blank control $(-)$. Trials were performed in a PBS environment using a 1:8 $\mathrm{Dap}_{\mathrm{Ca}^{2+}}$ ratio. The individual trials are shown as $\times$. The averages for the curve fits are shown as $\circ$.

binds to its outer membrane (Fig. 4,-). Prior studies have suggested that the lack of daptomycin activity could be due to the drug's inability to either interact with or disrupt the cytoplasmic membrane. ${ }^{99}$ Our results unequivocally show that the former suggestion is not the case. Further, our results are supported by a study by Faust et al. that revealed daptomycin was capable of permeating model spheroplast vesicles consisting of phospholipid species extracted from the cytoplasmic membrane of E. coli cells. ${ }^{102}$

The daptomycin binding affinities appear to be similar for all of the bacterial species with the extracted $K_{d}$ averages shown Table 1. Fig. 4 reveals that E. faecalis (-) yielded Langmuir-shaped curves with higher $I_{S H G}$ in comparison to the gram-negative $E$. coli $(-)$. The lower intensities for E. coli can be explained by a lower number of binding sites for daptomycin as this species contains approximately $\sim 30 \%$ PG phospholipids in its phospholipidmembrane composition ${ }^{103-105}$ as opposed to the combined $\sim 38 \%$ PG and $\sim 42 \%$ CL for E. faecalis. ${ }^{106}$

S. aureus cells produce the lowest intensity SHG for the daptomycin isotherm (Fig. 4,一). The intensity is still greater than that exhibited in the blank control sample demonstrating 
clear daptomycin binding which is consistent with the fact that daptomycin is known to be active against this gram-positive species. ${ }^{7,8,29,107-109}$ In addition, the relative amount of negative phospholipids in the $S$. aureus membranes is similar to that of E. faecalis. ${ }^{15}$ Instead, we expect that the lower SHG signal arising from daptomycin binding to S. aureus membranes arises from larger fluctuations in the molecular alignment. The lipid composition of $S$. aureus has been shown to contain a higher percentage of branched fatty acids ${ }^{110,111}$ leading to an overall higher fluidity. ${ }^{112}$ When the molecule embeds into such a membrane, the fluidity will allow for it to sample a larger range of angles reducing the overall average orientation and thus the SHG intensity.

\section{Conclusions}

The work presented here includes the first reports for native daptomycin binding affinity constants with living bacterial membranes under different environmental conditions. These efforts were realized through the use of SHG spectroscopy which showcases its capabilities for quantifying small molecule interactions in living biological systems label-free. In doing so, the presence of free phosphate ions in the PBS environment were revealed to participate in the formation of a large and unique daptomycin- $\mathrm{Ca}^{2+}$ complexes. This phosphate-induced daptomycin- $\mathrm{Ca}^{2+}$ complex also yielded adsorption signals with larger intensities upon binding to E. faecalis membranes in comparison to the phosphate-free environment. We can conclude then that the environmental phosphate ions alter the daptomycin binding and needs to be considered in future proposed mechanisms for this drug. The $K_{d}$ values for multiple bacterial species were also measured which revealed that daptomycin has similar binding affinities for both the gram-positive and gram-negative bacteria studied. While daptomycin is known to not be effective against the gram-negative E. coli, ${ }^{7,8,99}$ the SHG daptomycin adsorption isotherms clearly show that daptomycin does bind to E. coli membranes. Overall, our SHG studies have provided new insight into the factors affecting the initial binding of 
daptomycin to living cells.

\section{Methods}

\section{Bacterial Cultures}

For testing the gram-positive bacterial species, single colonies of E. faecalis (OG1RF) and S. aureus (ATCC 27217) were grown on brain heart infusion (BHI) agar plates and inoculated into BHI liquid medium with a 1:5 liquid-to-head space volume ratio. The liquid cultures were statically grown overnight at $37^{\circ} \mathrm{C}$. For the gram-negative bacterial studies, single colonies of E. coli (K-12) were taken from Luria broth (LB) agar plates and inoculated into LB medium with a 1:5 liquid-to-head space volume ratio and grown overnight to stationary phase while shaking at 250 revolutions per minute at $37^{\circ} \mathrm{C}$. Once the cultures reached stationary phase, the cells were diluted into either fresh BHI or LB media to an optical density at $600 \mathrm{~nm}\left(\mathrm{OD}_{600}\right)$ of $\sim 0.01$ and grown under the same overnight growth parameters until reaching an $\mathrm{OD}_{600}$ of $\sim 0.3$. The bacterial cultures were washed twice via vacuum filtration with either sterile $\mathrm{PBS}$ or sterile $0.9 \% \mathrm{NaCl}$, dependent on the experimental isotherm saline conditions being tested. The cells were then resuspended in either PBS or $0.9 \% \mathrm{NaCl}$ saline, each containing $2 \%$ OxyRase, to a final $\mathrm{OD}_{600} \sim 0.3$. The final cell densities were measured using the UV-Vis spectrometer before performing the isotherm trials.

\section{Drug Solution Prep for Daptomycin-Dependent Isotherms}

For the daptomycin concentration-dependent isotherm trials, the daptomycin stock solutions were prepared using either the $\mathrm{Ca}^{2+}$ or $\mathrm{Mg}^{2+}$ ions. The dicationic stocks were made by dissolving calcium chloride or magnesium chloride in an 80:20 ratio of MilliQ deionized water $\left(\mathrm{H}_{2} \mathrm{O}\right)$ :dimethylsulfoxide (DMSO) to a final concentration of $800 \mathrm{mM}$. The $\mathrm{X}^{2+}$ (where $\mathrm{X}^{2+}=$ $\mathrm{Ca}^{2+}$ or $\mathrm{Mg}^{2+}$ ) ionic stock solutions were each filter-sterilized using 0.2 micron syringe filters. 
For maintaining a constant 1:8 Dap: $\mathrm{X}^{2+}$ ion ratio for at each of the different daptomycin concentrations studied for the isotherms, a $100 \mathrm{mM}$ daptomycin stock solution was prepared using the $800 \mathrm{mM} \mathrm{X}^{2+}$ ion solutions as the solvent. Additional 1:8 Dap: $\mathrm{X}^{2+}$ ion stock solutions were made by performing serial dilutions using sterile 80:20 $\mathrm{H}_{2} \mathrm{O}$ :DMSO as the solvent to achieve daptomycin stock solutions with concentrations ranging from $0 \mathrm{mM}$ to $100 \mathrm{mM}$. The daptomycin stock solutions were freshly prepared each day immediately before the isotherm experiments were conducted.

\section{Daptomycin Concentration-Dependent Isotherms}

Approximately $990 \mu \mathrm{L}$ of the prepared bacterial culture solutions from Section were aliquoted into sterile microcentrifuge vials and $10 \mu \mathrm{L}$ of the 1:8 Dap: $\mathrm{X}^{2+}$ solution corresponding to the appropriate drug concentration was added to the vials immediately before each drug concentration SHG measurement was conducted. The final DMSO percentage was held constant at $0.2 \%$ for each isotherm sample.

\section{Sample Cell Preparation}

A quartz flow cell, with a $2 \mathrm{~mm}$ path length, was passified using a bovine serum albumin (BSA) - 1\% glutaraldehyde crosslinking protocol ${ }^{113}$ and flushed with Tris buffer (pH adjusted using $\mathrm{HCl}$ to $\sim 7.4 \pm 0.1$ ) prior to the experiments. Approximately 20-30 minutes before the bacterial cells reached the appropriate cell density, a solution of $50 \mathrm{mM}$ BSA was prepared in cold $\left(4^{\circ} \mathrm{C}\right) 10 \mathrm{mM}$ Tris buffer and filter-sterilized using a 0.2 micron syringe filter. The BSA solution was introduced into the flow cell where it was allowed to incubate for approximately 10 minutes. Cold $10 \mathrm{mM}$ Tris buffer containing $1 \%$ glutaraldehyde was introduced to the flow cell following the BSA incubation period and allowed to incubate for approximately 10 minutes. Finally, $10 \mathrm{mM}$ Tris buffer was used to flush out the $1 \%$ glutaraldehyde solution from the flow cell. About three $1 \mathrm{~mL}$ aliquots of the prepped bacterial solutions were micropipetted into the flow cell and discarded to help remove the remaining Tris buffer in 
the flow cell before the SHG measurements were taken.

\section{SHG Spectroscopy Apparatus}

SHG signals were collected using a home-built spectroscopy instrument previously reported

elsewhere. ${ }^{42}$ Briefly, the $80 \mathrm{MHz}$ output of a MaiTai titanium:sapphire oscillator (SpectraPhysics) with pulse widths compressed to $\sim 80$ fs and centered at $800 \mathrm{~nm}$ was used to excite the sample. The average power at the sample position was adjusted to be $\sim 350 \mathrm{~mW}$ for all of the experiments. The SHG signals, centered at $400 \mathrm{~nm}$ were isolated with a series of optical filters before being detected with a photon-counting PMT. The SHG signals were collected every $250 \mathrm{~ms}$ and averaged over $3 \mathrm{~s}$.

Given that previous SHG bacterial membrane studies showed that a molecule smaller than daptomycin with no significant membrane association took approximately $\sim 28$ seconds

to cross the outer membrane of E. coli ${ }^{64}$ we do not expect significant internalization of the daptomycin within the $3 \mathrm{~s}$ time frame for the measurement of each data point. Total run time for each full SHG isotherm data set was approximately $\sim 20$ min. Each experimental condition was replicated with the number of trials reported as $n$ in Table 1.

\section{Data Processing}

For a given set of experimental conditions, each SHG isotherm trial was scaled to the average value of $0 \mu \mathrm{M}$ concentration SHG signal arising from HRS. The scaled intensities for each of the trial were averaged together, before fitting to the Langmuir equation (Eq. 1) using Matlab. The average $K_{d}$ constants and standard deviations were extracted from the fit parameters. 


\section{Dynamic Light Scattering}

Dynamic light scattering (DLS) measurements were taken with a Zetasizer Nano ZS to determine the daptomycin- $\mathrm{Ca}^{2+}$ aggregate diameter size of various Dap:Ca ${ }^{2+}$ ratios in sterile solutions consisting of $\mathrm{DI} \mathrm{H}_{2} \mathrm{O}, 0.9 \% \mathrm{NaCl}$ saline and PBS. Daptomycin concentration was held constant at $1 \mathrm{mM}$ for all of the trials.

\section{Acknowledgement}

The authors thank Elizabeth Fozo in the Department of Microbiology at the University of Tennessee, Knoxville for providing the bacterial strains. This work was funded by NIH/NIAID grant R01AI116571.

\section{Supporting Information Available}

Supporting Information is available free of charge:

- Expanded description of SHG

- Figures S1 and S2

\section{References}

(1) Organization, W. H. Urgent Health Challenges for the next Decade. https://www.who.int/news-room/photo-story/photo-story-detail/urgent-healthchallenges-for-the-next-decade, 2020.

(2) CDC, Antibiotic Resistance Threats in the United States; U.S. Department of Health and Human Services Report, 2019. 
(3) Mühlberg, E.; Umstätter, F.; Kleist, C.; Domhan, C.; Mier, W.; Uhl, P. Renaissance of Vancomycin: Approaches for Breaking Antibiotic Resistance in Multidrug-Resistant Bacteria. Canadian Journal of Microbiology 2019, 66, 11-16.

(4) Subramaniam, G.; Girish, M. Antibiotic Resistance - A Cause for Reemergence of Infections. The Indian Journal of Pediatrics 2020,

(5) Ventola, C. L. The Antibiotic Resistance Crisis: Part 1: Causes and Threats. Pharmacy and Therapeutics 2015, 40, 277-283.

(6) Jabbari Shiadeh, S. M.; Pormohammad, A.; Hashemi, A.; Lak, P. Global Prevalence of Antibiotic Resistance in Blood-Isolated Enterococcus Faecalis and Enterococcus Faecium: A Systematic Review and Meta-Analysis. Infection and drug resistance 2019, 12, 2713-2725.

(7) Tally, F. P.; Zeckel, M.; Wasilewski, M. M.; Carini, C.; Berman, C. L.; Drusano, G. L.; Oleson Jr, F. B. Daptomycin: A Novel Agent for Gram-Positive Infections. Expert Opinion on Investigational Drugs 1999, 8, 1223-1238.

(8) Carpenter, C. F.; Chambers, H. F. Daptomycin: Another Novel Agent for Treating Infections Due to Drug-Resistant Gram-Positive Pathogens. Clinical Infectious Diseases 2004, 38, 994-1000.

(9) Gray, A. D.; Wenzel, M. More than a Pore: A Current Perspective on the in Vivo Mode of Action of the Lipopeptide Antibiotic Daptomycin. Antibiotics 2020, 9.

(10) Ribeiro da Cunha, B.; Fonseca, L. P.; Calado, C. R. C. Antibiotic Discovery: Where Have We Come from, Where Do We Go? Antibiotics (Basel, Switzerland) 2019, 8, 45.

(11) Taylor, S. D.; Palmer, M. The Action Mechanism of Daptomycin. Bioorganic E Medicinal Chemistry 2016, 
(12) Heidary, M.; Khosravi, A. D.; Khoshnood, S.; Nasiri, M. J.; Soleimani, S.; Goudarzi, M. Daptomycin. Journal of Antimicrobial Chemotherapy 2017, 73, 1-11.

(13) Humphries, R. M.; Pollett, S.; Sakoulas, G. A Current Perspective on Daptomycin for the Clinical Microbiologist. Clinical Microbiology Reviews 2013, 26, 759-780.

(14) Werth, B. J.; Steed, M. E.; Ireland, C. E.; Tran, T. T.; Nonejuie, P.; Murray, B. E.; Rose, W. E.; Sakoulas, G.; Pogliano, J.; Arias, C. A.; Rybak, M. J. Defining Daptomycin Resistance Prevention Exposures in Vancomycin-Resistant Enterococcus Faecium and e. Faecalis. Antimicrobial Agents and Chemotherapy 2014, 58, 5253-5261.

(15) Mishra, N. N.; Yang, S.-J.; Sawa, A.; Rubio, A.; Nast, C. C.; Yeaman, M. R.; Bayer, A. S. Analysis of Cell Membrane Characteristics of in Vitro-Selected Daptomycin-Resistant Strains of Methicillin-Resistant Staphylococcus Aureus. Antimicrobial Agents and Chemotherapy 2009, 53, 2312-2318.

(16) Pogliano, J.; Pogliano, N.; Silverman, J. A. Daptomycin-Mediated Reorganization of Membrane Architecture Causes Mislocalization of Essential Cell Division Proteins. Journal of Bacteriology 2012, 194, 4494-4504.

(17) Tran, T. T.; Panesso, D.; Mishra, N. N.; Mileykovskaya, E.; Guan, Z.; Munita, J. M.; Reyes, J.; Diaz, L.; Weinstock, G. M.; Murray, B. E.; Shamoo, Y.; Dowhan, W.; Bayer, A. S.; Arias, C. A. Daptomycin-Resistant Enterococcus Faecalis Diverts the Antibiotic Molecule from the Division Septum and Remodels Cell Membrane Phospholipids. American Society for MicroBiology 2013, 4, 1-10.

(18) Mishra, N. N.; Bayer, A. S.; Tran, T. T.; Shamoo, Y.; Mileykovskaya, E.; Dowhan, W.; Guan, Z.; Arias, C. A. Daptomycin Resistance in Enterococci Is Associated with Distinct Alterations of Cell Membrane Phospholipid Content. PLoS ONE 2012, \%, e43958. 
(19) Tran, T. T.; Munita, J. M.; Arias, C. A. Mechanisms of Drug Resistance: Daptomycin Resistance. Annals of the New York Academy of Sciences 2015, 1354, 32-53.

(20) Bayer, A. S.; Schneider, T.; Sahl, H.-G. Mechanisms of Daptomycin Resistance in Staphylococcus Aureus: Role of the Cell Membrane and Cell Wall. Annals of the New York Academy of Sciences 2013, 1277, 139-158.

(21) Miller, W. R.; Bayer, A. S.; Arias, C. A. Mechanism of Action and Resistance to Daptomycin in Staphylococcus Aureus and Enterococci. Cold Spring Harbor perspectives in medicine 2016, 6, a026997.

(22) Hachmann, A.-B.; Sevim, E.; Gaballa, A.; Popham, D. L.; Antelmann, H.; Helmann, J. D. Reduction in Membrane Phosphatidylglycerol Content Leads to Daptomycin Resistance in Bacillus Subtilis. Antimicrobial Agents and Chemotherapy 2011, $55,4326-4337$.

(23) Taylor, R.; Butt, K.; Scott, B.; Zhang, T.; Muraih, J. K.; Mintzer, E.; Taylor, S.; Palmer, M. Two Successive Calcium-Dependent Transitions Mediate Membrane Binding and Oligomerization of Daptomycin and the Related Antibiotic A54145. Biochimica et Biophysica Acta (BBA) - Biomembranes 2016, 1858, 1999-2005.

(24) Muraih, J. K.; Pearson, A.; Silverman, J.; Palmer, M. Oligomerization of Daptomycin on Membranes. Biochimica et Biophysica Acta (BBA) - Biomembranes 2011, 1808, 1154-1160.

(25) Lakey, J. H.; Ptak, M. Fluorescence Indicates a Calcium-Dependent Interaction between the Lipopeptide Antibiotic LY 146032 and Phospholipid Membranes. Biochemistry 1988, 27, 4639-4645.

(26) Strieker, M.; Marahiel, M. A. The Structural Diversity of Acidic Lipopeptide Antibiotics. ChemBioChem 2009, 10, 607-616. 
(27) Ho, S. W.; Jung, D.; Calhoun, J. R.; Lear, J. D.; Okon, M.; Scott, W. R. P.; Hancock, R. E. W.; Straus, S. K. Effect of Divalent Cations on the Structure of the Antibiotic Daptomycin. European Biophysics Journal 2008, 37, 421-433.

(28) Scott, W. R. P.; Baek, S.-B.; Jung, D.; Hancock, R. E. W.; Straus, S. K. NMR Structural Studies of the Antibiotic Lipopeptide Daptomycin in DHPC Micelles. Biochimica et Biophysica Acta (BBA) - Biomembranes 2007, 1768, 3116-3126.

(29) Straus, S. K.; Hancock, R. E. W. Mode of Action of the New Antibiotic for GramPositive Pathogens Daptomycin: Comparison with Cationic Antimicrobial Peptides and Lipopeptides. Biochimica et Biophysica Acta (BBA) - Biomembranes 2006, 1758, $1215-1223$.

(30) Rotondi, K. S.; Gierasch, L. M. A Well-Defined Amphipathic Conformation for the Calcium-Free Cyclic Lipopeptide Antibiotic, Daptomycin, in Aqueous Solution. Peptide Science 2005, 80, 374-385.

(31) Ball, L.-J.; Goult, C. M.; Donarski, J. A.; Micklefield, J.; Ramesh, V. NMR Structure Determination and Calcium Binding Effects of Lipopeptide Antibiotic Daptomycin. Organic ${ }^{6} 3$ Biomolecular Chemistry 2004, 2, 1872-1878.

(32) Bunkóczi, G.; Vértesy, L.; Sheldrick, G. M. Structure of the Lipopeptide Antibiotic Tsushimycin. Acta Crystallographica Section D 2005, 61, 1160-1164.

(33) Grein, F.; Müller, A.; Scherer, K. M.; Liu, X.; Ludwig, K. C.; Klöckner, A.; Strach, M.; Sahl, H.-G.; Kubitscheck, U.; Schneider, T. Ca 2+-Daptomycin Targets Cell Wall Biosynthesis by Forming a Tripartite Complex with Undecaprenyl-Coupled Intermediates and Membrane Lipids. Nature Communications 2020, 11, 1455.

(34) Zhang, T.; Muraih, J. K.; MacCormick, B.; Silverman, J.; Palmer, M. Daptomycin Forms Cation- and Size-Selective Pores in Model Membranes. Biochimica et Biophysica Acta (BBA) - Biomembranes 2014, 1838, 2425-2430. 
(35) Jung, D.; Rozek, A.; Okon, M.; Hancock, R. E. W. Structural Transitions as Determinants of the Action of the Calcium-Dependent Antibiotic Daptomycin. Chemistry E Biology 2004, 11, 949-957.

(36) Jung, D.; Powers, J. P.; Straus, S. K.; Hancock, R. E. W. Lipid-Specific Binding of the Calcium-Dependent Antibiotic Daptomycin Leads to Changes in Lipid Polymorphism of Model Membranes. Chemistry and Physics of Lipids 2008, 154, 120-128.

(37) Pokorny, A.; Khatib, T. O.; Stevenson, H. A Quantitative Model of Daptomycin Binding to Lipid Bilayers. The Journal of Physical Chemistry B 2018, 122, 91379146.

(38) Qiu, J.; Kirsch, L. E. Evaluation of Lipopeptide (Daptomycin) Aggregation Using Fluorescence, Light Scattering, and Nuclear Magnetic Resonance Spectroscopy. Journal of Pharmaceutical Sciences 2014, 103, 853-861.

(39) Zhang, J.; Scoten, K.; Straus, S. K. Daptomycin Leakage Is Selective. ACS Infectious Diseases 2016, 2, 682-687.

(40) Zhang, J.; Scott, W. R. P.; Gabel, F.; Wu, M.; Desmond, R.; Bae, J.; Zaccai, G.; Algar, W. R.; Straus, S. K. On the Quest for the Elusive Mechanism of Action of Daptomycin: Binding, Fusion, and Oligomerization. Biochimica et Biophysica Acta (BBA) - Proteins and Proteomics 2017, 1865, 1490-1499.

(41) Okur, H. I.; Tarun, O. B.; Roke, S. Chemistry of Lipid Membranes from Models to Living Systems: A Perspective of Hydration, Surface Potential, Curvature, Confinement and Heterogeneity. J. Am. Chem. Soc. 2019, 141, 12168-12181.

(42) Miller, L. N.; Brewer, W. T.; Williams, J. D.; Fozo, E. M.; Calhoun, T. R. Second Harmonic Generation Spectroscopy of Membrane Probe Dynamics in Gram-Positive Bacteria. Biophysical Journal 2019, 117, 1419-1428. 
(43) Doerr, A. Imaging Goes Label-Free. Nature Methods 2009, 6, 116.

(44) Stewart, P. S.; Davison, W. M.; Steenbergen, J. N. Daptomycin Rapidly Penetrates a Staphylococcus Epidermidis Biofilm. Antimicrobial Agents and Chemotherapy 2009, 53, 3505-3507.

(45) Zipfel, W. R.; Williams, R. M.; Webb, W. W. Nonlinear Magic: Multiphoton Microscopy in the Biosciences. Nature Biotechnology 2003, 21, 1369-1377.

(46) Torno, K.; Wright, B. K.; Jones, M. R.; Digman, M. A.; Gratton, E.; Phillips, M. Real-Time Analysis of Metabolic Activity Within Lactobacillus Acidophilus by Phasor Fluorescence Lifetime Imaging Microscopy of NADH. Curr Microbiol 2013, 66, 365367.

(47) Doughty, B.; Rao, Y.; Kazer, S. W.; Kwok, S. J. J.; Turro, N. J.; Eisenthal, K. B. Binding of the Anti-Cancer Drug Daunomycin to DNA Probed by Second Harmonic Generation. The Journal of Physical Chemistry B 2013, 117, 15285-15289.

(48) Liu, J.; Shang, X.; Pompano, R.; Eisenthal, K. B. Antibiotic Assisted Molecular Ion Transport across a Membrane in Real Time. Faraday Discussions 2005, 129, 291-299.

(49) Liu, Y.; Yan, E. C. Y.; Eisenthal, K. B. Effects of Bilayer Surface Charge Density on Molecular Adsorption and Transport across Liposome Bilayers. Biophysical Journal 2001, 80, 1004-1012.

(50) Liu, J.; Subir, M.; Nguyen, K.; Eisenthal, K. B. Second Harmonic Studies of Ions Crossing Liposome Membranes in Real Time. The Journal of Physical Chemistry B 2008, 112, 15263-15266.

(51) Liu, Y.; Yan, E. C. Y.; Zhao, X.; Eisenthal, K. B. Surface Potential of Charged Liposomes Determined by Second Harmonic Generation. Langmuir 2001, 17, 20632066. 
(52) Salafsky, J. S. Second-Harmonic Generation as a Probe of Conformational Change in Molecules. Chemical Physics Letters 2003, 381, 705-709.

(53) Shang, X.; Liu, Y.; Yan, E.; Eisenthal, K. B. Effects of Counterions on Molecular Transport across Liposome Bilayer: Probed by Second Harmonic Generation. The Journal of Physical Chemistry B 2001, 105, 12816-12822.

(54) Srivastava, A.; Eisenthal, K. B. Kinetics of Molecular Transport across a Liposome Bilayer. Chemical Physics Letters 1998, 292, 345-351.

(55) Blanchard-Desce, M. H.; Ventelon, L.; Charier, S.; Moreaux, L.; Mertz, J. Molecular Probes for Nonlinear Optical Imaging of Biological Membranes. Proc. SPIE, Linear and Nonlinear Optics of Organic Materials 2001, 4461, 20-32.

(56) Mertz, J. Applications of Second-Harmonic Generation Microscopy. 2008, 348-376.

(57) Moreaux, L.; Sandre, O.; Charpak, S.; Blanchard-Desce, M.; Mertz, J. Coherent Scattering in Multi-Harmonic Light Microscopy. Biophysical Journal 2001, 80, 1568-1574.

(58) Moreaux, L.; Sandre, O.; Blanchard-Desce, M.; Mertz, J. Membrane Imaging by Simultaneous Second-Harmonic Generation and Two-Photon Microscopy. Optics Letters 2000, 25, 320-322.

(59) Pons, T.; Moreaux, L.; Mertz, J. Photoinduced Flip-Flop of Amphiphilic Molecules in Lipid Bilayer Membranes. Physical Review Letters 2002, 89, 288104.

(60) Sharifian Gh, M.; Wilhelm, M. J.; Dai, H.-L. Label-Free Optical Method for Quantifying Molecular Transport across Cellular Membranes in Vitro. The Journal of Physical Chemistry Letters 2016, 7, 3406-3411.

(61) Wilhelm, M. J.; Sharifian Gh, M.; Dai, H.-L. Chemically Induced Changes to Membrane Permeability in Living Cells Probed with Nonlinear Light Scattering. Biochemistry 2015, 54, 4427-4430. 
(62) Wilhelm, M. J.; Sheffield, J. B.; Sharifian Gh, M.; Wu, Y.; Spahr, C.; Gonella, G.; Xu, B.; Dai, H.-L. Gram's Stain Does Not Cross the Bacterial Cytoplasmic Membrane. ACS Chemical Biology 2015, 10, 1711-1717.

(63) Wilhelm, M. J.; Sheffield, J. B.; Gonella, G.; Wu, Y.; Spahr, C.; Zeng, J.; Xu, B.; Dai, H.-L. Real-Time Molecular Uptake and Membrane-Specific Transport in Living Cells by Optical Microscopy and Nonlinear Light Scattering. Chemical Physics Letters 2014, 605-606, 158-163.

(64) Zeng, J.; Eckenrode, H. M.; Dounce, S. M.; Dai, H.-L. Time-Resolved Molecular Transport across Living Cell Membranes. Biophysical Journal 2013, 104, 139-145.

(65) Zeng, J.; Eckenrode, H. M.; Dai, H.-L.; Wilhelm, M. J. Adsorption and Transport of Charged vs. Neutral Hydrophobic Molecules at the Membrane of Murine Erythroleukemia (MEL) Cells. Colloids and Surfaces B: Biointerfaces 2015, 127, 122-129.

(66) Nuriya, M.; Fukushima, S.; Momotake, A.; Shinotsuka, T.; Yasui, M.; Arai, T. Multimodal Two-Photon Imaging Using a Second Harmonic Generation-Specific Dye. Nature communications 2016, 7, 11557-11557.

(67) Gayen, A.; Kumar, D.; Matheshwaran, S.; Chandra, M. Unveiling the Modulating Role of Extracellular pH in Permeation and Accumulation of Small Molecules in Subcellular Compartments of Gram-Negative Escherichia Coli Using Nonlinear Spectroscopy. Analytical Chemistry 2019,

(68) Dombeck, D. A.; Sacconi, L.; Blanchard-Desce, M.; Webb, W. W. Optical Recording of Fast Neuronal Membrane Potential Transients in Acute Mammalian Brain Slices by Second-Harmonic Generation Microscopy. Journal of Neurophysiology 2005, 94, 3628-3636.

(69) Eisenthal, K. B. Second Harmonic Spectroscopy of Aqueous Nano- and Microparticle Interfaces. Chem. Rev. 2006, 106, 1462-1477. 
(70) Kinouchi, H.; Onishi, M.; Kamimori, H. Lipid Membrane-Binding Properties of Daptomycin Using Surface Plasmon Resonance. Analytical Sciences 2013, 29, 297-301.

(71) Lee, M.-T.; Hung, W.-C.; Hsieh, M.-H.; Chen, H.; Chang, Y.-Y.; Huang, H. W. Molecular State of the Membrane-Active Antibiotic Daptomycin. Biophysical Journal 2017, 113, 82-90.

(72) Muraih, J. K.; Harris, J.; Taylor, S. D.; Palmer, M. Characterization of Daptomycin Oligomerization with Perylene Excimer Fluorescence: Stoichiometric Binding of Phosphatidylglycerol Triggers Oligomer Formation. Biochimica et Biophysica Acta (BBA) - Biomembranes 2012, 1818, 673-678.

(73) Muraih, J. K.; Palmer, M. Estimation of the Subunit Stoichiometry of the MembraneAssociated Daptomycin Oligomer by FRET. Biochimica et Biophysica Acta (BBA) Biomembranes 2012, 1818, 1642-1647.

(74) Nguyen, T. T.; Conboy, J. C. High-Throughput Screening of Drug-Lipid Membrane Interactions via Counter-Propagating Second Harmonic Generation Imaging. Analytical Chemistry 2011, 83, 5979-5988.

(75) Kriech, M. A.; Conboy, J. C. Label-Free Chiral Detection of Melittin Binding to a Membrane. Journal of the American Chemical Society 2003, 125, 1148-1149.

(76) Petralli-Mallow, T. P.; Plant, A. L.; Lewis, M. L.; Hicks, J. M. Cytochrome c at Model Membrane Surfaces: Exploration via Second Harmonic Generation-Circular Dichroism and Surface-Enhanced Resonance Raman Spectroscopy. Langmuir 2000, $16,5960-5966$.

(77) Kriech, J. C. C.; A., M. Measuring Melittin Binding to Planar Supported Lipid Bilayer by Chiral Second Harmonic Generation. Analytica Chimica Acta 2003, 496, 143-153. 
(78) Salafsky, J. S.; Eisenthal, K. B. Second Harmonic Spectroscopy: Detection and Orientation of Molecules at a Biomembrane Interface. Chemical Physics Letters 2000, 319, $435-439$.

(79) Rao, Y.; Kwok, S. J. J.; Lombardi, J.; Turro, N. J.; Eisenthal, K. B. Label-Free Probe of HIV-1 TAT Peptide Binding to Mimetic Membranes. Proceedings of the National Academy of Sciences 2014, 111, 12684-12688.

(80) Tran, R. J.; Sly, K. L.; Conboy, J. C. Applications of Surface Second Harmonic Generation in Biological Sensing. Annual Review of Analytical Chemistry 2017, 10, 387-414.

(81) Stokes, G. Y.; Conboy, J. C. Measuring Selective Estrogen Receptor Modulator (SERM)-Membrane Interactions with Second Harmonic Generation. Journal of the American Chemical Society 2014, 136, 1409-1417.

(82) Kumal, R. R.; Nguyenhuu, H.; Winter, J. E.; McCarley, R. L.; Haber, L. H. Impacts of Salt, Buffer, and Lipid Nature on Molecular Adsorption and Transport in Liposomes as Observed by Second Harmonic Generation. The Journal of Physical Chemistry C 2017, 121, 15851-15860.

(83) Ehlert, F.; Neu, H. C. In Vitro Activity of LY146032 (Daptomycin), a New Peptolide. European Journal of Clinical Microbiology 1987, 6, 84-90.

(84) Tally, F. P.; DeBruin, M. F. Development of Daptomycin for Gram-Positive Infections. Journal of Antimicrobial Chemotherapy 2000, 46, 523-526.

(85) Chen, Y.-F.; Sun, T.-L.; Sun, Y.; Huang, H. W. Interaction of Daptomycin with Lipid Bilayers: A Lipid Extracting Effect. Biochemistry 2014, 53, 5384-5392.

(86) Harp, J. R.; Saito, H. E.; Bourdon, A. K.; Reyes, J.; Arias, C. A.; Campagna, S. R.; Fozo, E. M. Exogenous Fatty Acids Protect Enterococcus Faecalis from Daptomycin- 
Induced Membrane Stress Independently of the Response Regulator LiaR. Applied and Environmental Microbiology 2016, 82, 4410-4420.

(87) Saito, H. E.; Harp, J. R.; Fozo, E. M. Incorporation of Exogenous Fatty Acids Protects Enterococcus Faecalis from Membrane-Damaging Agents. Applied and environmental microbiology 2014, 80, 6527-6538.

(88) Morvan, C.; Halpern, D.; Kénanian, G.; Hays, C.; Anba-Mondoloni, J.; Brinster, S.; Kennedy, S.; Trieu-Cuot, P.; Poyart, C.; Lamberet, G.; Gloux, K.; Gruss, A. Environmental Fatty Acids Enable Emergence of Infectious Staphylococcus Aureus Resistant to FASII-Targeted Antimicrobials. Nature Communications 2016, 7, 12944.

(89) Boudjemaa, R.; Cabriel, C.; Dubois-Brissonnet, F.; Bourg, N.; Dupuis, G.; Gruss, A.; Lévêque-Fort, S.; Briandet, R.; Fontaine-Aupart, M.-P.; Steenkeste, K. Impact of Bacterial Membrane Fatty Acid Composition on the Failure of Daptomycin to Kill Staphylococcus Aureus. Antimicrobial agents and chemotherapy 2018, 62, e0002318.

(90) Lo Nostro, P.; Ninham, B. W. Hofmeister Phenomena: An Update on Ion Specificity in Biology. Chemical Reviews 2012, 112, 2286-2322.

(91) Ninham, B. W.; Lo Nostro, P. Molecular Forces and Self Assembly: In Colloid, Nano Sciences and Biology; Cambridge Molecular Science; Cambridge University Press: Cambridge, 2010.

(92) Leray, A.; Rouède, D.; Odin, C.; Grand, Y. L.; Mongin, O.; Blanchard-Desce, M. Effect of the Orientational Disorder on the Hyperpolarizability Measurement of Amphiphilic Push-Pull Chromophores in Langmuir-Blodgett Monolayers. Optics Communications 2005, 247, 213-223.

(93) Himeno, H.; Shimokawa, N.; Komura, S.; Andelman, D.; Hamada, T.; Takagi, M. 
Charge-Induced Phase Separation in Lipid Membranes. Soft Matter 2014, 10, 7959 7967.

(94) Yang, N. J.; Hinner, M. J. Getting across the Cell Membrane: An Overview for Small Molecules, Peptides, and Proteins. Methods in molecular biology (Clifton, N.J.) 2015, 1266, 29-53.

(95) Cheng, J. T. J.; Hale, J. D.; Elliott, M.; Hancock, R. E. W.; Straus, S. K. The Importance of Bacterial Membrane Composition in the Structure and Function of Aurein 2.2 and Selected Variants. Biochimica et Biophysica Acta (BBA) - Biomembranes 2011, 1808, 622-633.

(96) Delcour, A. H. Outer Membrane Permeability and Antibiotic Resistance. Biochimica et Biophysica Acta (BBA) - Proteins and Proteomics 2009, 1794, 808-816.

(97) Epand, R. M.; Epand, R. F. Bacterial Membrane Lipids in the Action of Antimicrobial Agents. Journal of Peptide Science 2011, 17, 298-305.

(98) Epand, R. M.; Walker, C.; Epand, R. F.; Magarvey, N. A. Molecular Mechanisms of Membrane Targeting Antibiotics. Biochimica et Biophysica Acta (BBA) - Biomembranes 2016, 1858, 980-987.

(99) Randall, C. P.; Mariner, K. R.; Chopra, I.; O’Neill, A. J. The Target of Daptomycin Is Absent from Escherichia Coli and Other Gram-Negative Pathogens. Antimicrobial Agents and Chemotherapy 2013, 57, 637-639.

(100) Rashid, R.; Cazenave-Gassiot, A.; Gao, I. H.; Nair, Z. J.; Kumar, J. K.; Gao, L.; Kline, K. A.; Wenk, M. R. Comprehensive Analysis of Phospholipids and Glycolipids in the Opportunistic Pathogen Enterococcus Faecalis. PloS one 2017, 12, e0175886$\mathrm{e} 0175886$. 
(101) Zhang, Y.-M.; Rock, C. O. Membrane Lipid Homeostasis in Bacteria. Nat Rev Micro 2008, 6, 222-233.

(102) Faust, J. E.; Yang, P.-Y.; Huang, H. W. Action of Antimicrobial Peptides on Bacterial and Lipid Membranes: A Direct Comparison. Biophysical Journal 2017, 112, 16631672.

(103) Epand, R. F.; Savage, P. B.; Epand, R. M. Bacterial Lipid Composition and the Antimicrobial Efficacy of Cationic Steroid Compounds (Ceragenins). Biochimica et Biophysica Acta (BBA) - Biomembranes 2007, 1768, 2500-2509.

(104) Oliver, P. M.; Crooks, J. A.; Leidl, M.; Yoon, E. J.; Saghatelian, A.; Weibel, D. B. Localization of Anionic Phospholipids in Escherichia Coli Cells. Journal of Bacteriology 2014, 196, 3386-3398.

(105) Oursel, D.; Loutelier-Bourhis, C.; Orange, N.; Chevalier, S.; Norris, V.; Lange, C. M. Lipid Composition of Membranes of Escherichia Coli by Liquid Chromatography/Tandem Mass Spectrometry Using Negative Electrospray Ionization. Rapid Communications in Mass Spectrometry 2007, 21, 1721-1728.

(106) Khan, A. et al. Antimicrobial Sensing Coupled with Cell Membrane Remodeling Mediates Antibiotic Resistance and Virulence in Enterococcus Faecalis. Proc Natl Acad Sci USA 2019, 116, 26925.

(107) Tedesco, K. L.; Rybak, M. J. Daptomycin. Pharmacotherapy: The Journal of Human Pharmacology and Drug Therapy 2004, 24, 41-57.

(108) Fenton, C.; Fenton, C.; Keating, G. M.; Curran, M. P. Daptomycin. Drugs 2004, 64, $445-455$.

(109) Thorne, G. M.; Alder, J. Daptomycin: A Novel Lipopeptide Antibiotic. Clinical Microbiology Newsletter 2002, 24, 33-40. 
(110) Kumariya, R.; Sood, S. K.; Rajput, Y. S.; Saini, N.; Garsa, A. K. Increased Membrane Surface Positive Charge and Altered Membrane Fluidity Leads to Cationic Antimicrobial Peptide Resistance in Enterococcus Faecalis. Biochimica et Biophysica Acta (BBA) - Biomembranes 2015, 1848, 1367-1375.

(111) Sen, S.; Sirobhushanam, S.; Johnson, S. R.; Song, Y.; Tefft, R.; Gatto, C.; Wilkinson, B. J. Growth-Environment Dependent Modulation of Staphylococcus Aureus Branched-Chain to Straight-Chain Fatty Acid Ratio and Incorporation of Unsaturated Fatty Acids. PLOS ONE 2016, 11, e0165300.

(112) Mitchell, N. J.; Seaton, P.; Pokorny, A. Branched Phospholipids Render Lipid Vesicles More Susceptible to Membrane-Active Peptides. Biochimica et Biophysica Acta (BBA) - Biomembranes 2016, 1858, 988-994.

(113) Park, J. H.; Sut, T. N.; Jackman, J. A.; Ferhan, A. R.; Yoon, B. K.; Cho, N.-J. Controlling Adsorption and Passivation Properties of Bovine Serum Albumin on Silica Surfaces by Ionic Strength Modulation and Cross-Linking. Physical Chemistry Chemical Physics 2017, 19, 8854-8865. 


\section{TOC Graphic}

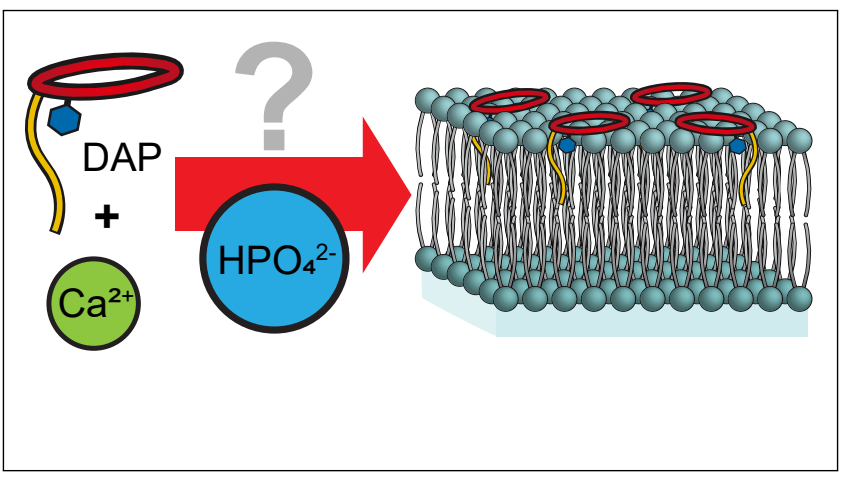

